\title{
Passive control of instability regions by means of piezoceramic elements
}

\section{Krzysztof Sokół ${ }^{\text {a* }}$}

a Politechnika Częstochowska, ul. J.H. Dąbrowskiego 69 42-201 Częstochowa, Poland. E-mail: sokol@imipkm.pcz.pl

${ }^{*}$ Corresponding author

https://doi.org/10.1590/1679-78256015

\begin{abstract}
The studies on vibration frequency as well as loading capacity of slender systems are very important issues in the design process. Integration of smart materials with the host structures allows one to create constructions which are able to adjust to the different conditions. The piezo material belongs to the smart materials group. Application of voltage to the piezoparts leads to induction of forces which can cause prestressing of the host structure. The prestressing with the use of piezoceramic elements is a main subject of this paper. The investigated system constitutes a column which is loaded with the specific load. The specific load is realized by means of specially designed heads what results in divergence or divergence-pseudoflutter type of characteristic curves. The results presented in this paper are concern on comparison of the loading capacity magnitude and vibration frequency of a slender system with and without prestressing. Finally, it will be stated when to use complex system with piezopart or the simplified one without it.
\end{abstract}

\section{Keywords}

Vibration control, piezo, instability, characteristic curve, slender system.

\section{Graphical Abstract}

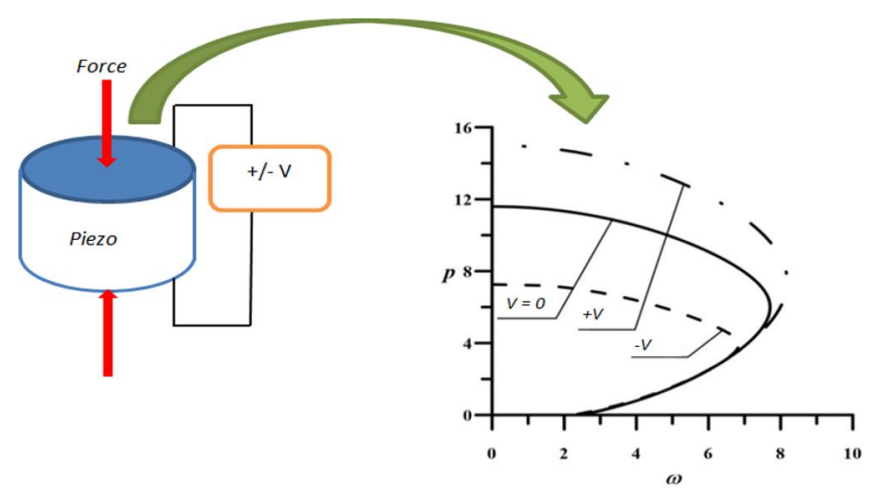




\section{INTRODUCTION}

One of the main problems in engineering are studies on vibrations and loading capacity of slender systems (Song et al. (2006)). Those studies are especially important when the system is subjected to static or dynamic excitations. The piezoceramic material that contains in the group of smart materials is a solution for this problem. The integration of piezoceramic sensors or actuators which are being produced in many different sizes and shapes (plates, discs, rods, tubes) may lead to the active or passive stability control.

As a result of studies realized by Thompson and Loughlan (1995), Irschik (2002) it was stated that in case of initially deformed structures integrated with piezoelements one may achieve the shape control phenomenon. Depending on installation of piezoelements in the host structure one can obtain bending or axial deformations. The shape control is not the only field where piezoelements can be used. The next one is active dynamic instability control. Studies in this direction were performed inter alia by Mukherjee and Saha Chaudhuri $(2002,2004)$.

Faria (2004) has investigated a problem of an increase of the buckling forces of beams with piezoelements. Sokół and Uzny (2015) have presented results of studies on an influence of the residual forces induced by piezoelements on the instability of the column subjected to Euler's load. Zenz and Humer (2015) have performed theoretical as well as numerical studies on piezoelements that shown an increase in loading capacity of an investigated structure. The phenomenon of instability control of columns was presented by Sokół (2019). Author used piezorods for prestresing of the structure.

Piezoelements have also been used as a part of composite plates (Adali et al., 2005). Their role was to strengthen a plate under given load. The studies on vibration of piezoplates were described by Kozupa and Wiciak (2010). As a result of an experiment the damping of vibrations has been achieved in every considered excitation type. Shakeri Mobarakeh et al. (2019) have studied the vibrations of parallelogram-shaped bimorph piezoceramic plates. As shown on the basis of the superposition authors have developed an effective method for analytical solution of the problem in relation to the classical solutions.

Bearing in mind that most of engineering structures have shape and assembly imperfection Przybylski and Sokół (2011) have recommended an eccentric installation of a piezoceramic part in order to control the defection of the system. After numerical simulations it was stated that, after the voltage has been applied to the piezoceramic rod, the host rod regained the rectilinear form of static equilibrium.

Yang et al. (2020) have integrated piezoelements with cantilever beams. Authors have used perturbation method to solve free damping vibrations problem. Finally in the numerical results one has discussed an influence of the piezoelectric properties on the vibration of piezoelectric cantilever beams. Peng et al. (2019) have studied time-delayed feedback control to reduce the nonlinear resonant vibration of a piezoelectric beam. They have achieved the control on stability, amplitude and frequency-response behavior. Wankhade and Bajoria (2017) studied the optimization phenomenon of beams with piezoelements which were subjected to the static and dynamic excitations. Scientists have found the optimal location of a piezoelectric patch for the chosen boundary condition of the system. The same authors two years later (Wankhade and Bajoria (2019)) have focused on vibrations of piezolaminated plates for sensing and actuating applications. The studied system was subjected to the dynamic excitation. Results have shown that the electromechanical coupling can strengthen the plate's resistance to vibration. Bendine and Wankhade (2017) used piezoceramic elements to control the shape control of beams with different boundary condition and loading.

The main purposes of this paper is to present an influence of the residual force generated by the piezorod on vibrations and loading capacity of a column subjected to the external specific load. The external load is being realized with heads with circular outline. The introduced type of external load leads to divergence or divergence - psedoflutter shape of the characteristic curve in relation to the parameters of heads. It is worth to mention that the piezoelectric prestressing of columns subjected to the specific load has not been previously presented in the scientific literature.

\section{BOUNDARY PROBLEM DESCRIPTION}

Figure 1 shows the investigated system which is subjected to the specific load. The structure of loading heads used in this study was presented by Tomski and Uzny (2013). 


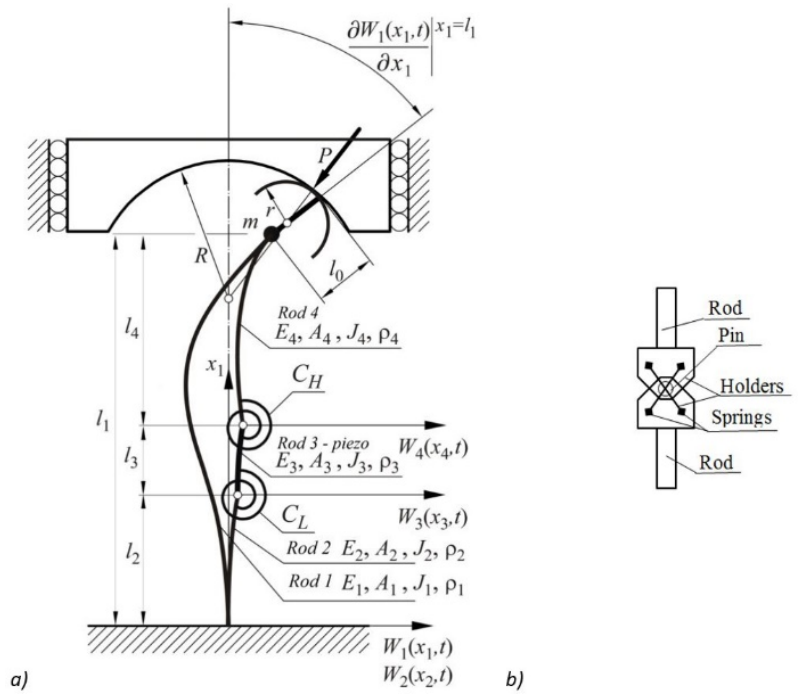

Figure 1: Investigated column - a) bent axes, b) connection of piezorod to the host structure.

The mathematical model is created on the basis of the following assumptions: the material of rods is homogeneous, the column has rectilinear form of static equilibrium, investigated system is a slender one what allows one to use Bernoulli - Euler theory, the support has an infinite stiffness, friction between elements in omitted.

Rod 1 is a single element and creates an external member / while rods 2, 3 and 4 are placed in an internal member II. Bearing in mind cost and production of piezorods it is proposed to use rod 3 as piezoceramic one. The connection of the piezorod to the host structure will be done by means of pins and rotational springs $C_{L}$ and $C_{H}$ what allows one to simulate different connection stiffness. In the real structure such a connection can be done inter alia as shown in the Figure $1 \mathrm{~b}$ where uses specially designed rod holders connected with a pin and springs. The natural boundary conditions satisfy the continuity of displacements, longitudinal forces, deflection angles as well as bending moments. Rods have length: $I_{1}, I_{2}, I_{3}, I_{4}-$ where $I=I_{1}=I_{2}+I_{3}+I_{4}$. The $m$ describes the mass of heads. The heads have radiuses $R$ and $r$ for loading head and load receiving one respectively. The radius of the loading head starts in the pole point located on the undeformed axis of the investigated slender system. The $I_{0}$ describes the distance between the end of the host structure and the contact point of heads.

In this paper at first with use of the Hamilton's principle (expression 1) one can find the differential equations of motion and natural boundary conditions. Later the mathematical formulation of the residual internal force generated by piezorod is presented. The mix of those solutions fully describes the studied problem.

$\delta \int_{t_{1}}^{t_{2}}(T-V) d t$

In the above expression $T$ presents the kinetic energy

$T=\frac{1}{2} \sum_{i=10}^{4} \int_{i}^{l_{i}} \rho_{i} A_{i}\left[\frac{\partial W_{i}\left(x_{i}, t\right)}{\partial t}\right]^{2} d x_{i}+\frac{1}{2} m\left[\left.\frac{\partial W_{i}\left(x_{i}, t\right)}{\partial t}\right|^{x_{i}=l_{i}}\right]^{2}$

while $V$ stands for the potential one 


$$
\begin{aligned}
& V=\frac{1}{2} \sum_{i=10}^{4} \int_{i}^{l_{i}} E_{i} J_{i}\left[\frac{\partial^{2} W_{i}\left(x_{i}, t\right)}{\partial x_{i}^{2}}\right]^{2} d x_{i}+\frac{1}{2} C_{L}\left(\left.\frac{\partial W_{3}\left(x_{3}, t\right)}{\partial x_{3}}\right|_{x_{3}=0}-\left.\frac{\partial W_{2}\left(x_{2}, t\right)}{\partial x_{2}}\right|^{x_{2}=l_{2}}\right)^{2}+ \\
& +\frac{1}{2} C_{H}\left(\left.\frac{\partial W_{4}\left(x_{4}, t\right)}{\partial x_{4}}\right|_{x_{4}=0}-\left.\frac{\partial W_{3}\left(x_{3}, t\right)}{\partial x_{3}}\right|^{x_{3}=l_{3}}\right)^{2}+ \\
& +\frac{1}{2} \sum_{i=10}^{4} \int_{i}^{l_{i}} E_{i} A_{i}\left[\frac{\partial U_{i}\left(x_{i}, t\right)}{\partial x_{i}}+\frac{1}{2}\left(\frac{\partial W_{i}\left(x_{i}, t\right)}{\partial x_{i}}\right)\right]^{2} d x_{i}+ \\
& +P U_{l}\left(l_{l}, t\right)-\frac{1}{2} P l_{0}\left(\left.\frac{\partial W_{l}\left(x_{l}, t\right)}{\partial x_{l}}\right|^{x_{l}=l_{l}}\right)^{2}+\frac{1}{2} \operatorname{Pr}\left\{\left[\left.\frac{\partial W_{l}\left(x_{l}, t\right)}{\partial x_{l}}\right|^{x_{l}=l_{l}}\right]^{2}-\chi^{2}\right\}+\frac{1}{2} P \chi W^{*} \\
& \chi=\frac{1}{R-r}\left(W_{l}\left(l_{1}, t\right)+\left.\left(l_{0}-r\right) \frac{\partial W_{1}\left(x_{1}, t\right)}{\partial x_{l}}\right|^{x_{1}=l_{l}}\right) \\
& W^{*}=\left.W_{l}\left(l_{l}, t\right) \frac{\partial W_{l}\left(x_{1}, t\right)}{\partial x_{1}}\right|^{x_{l}=l_{l}}\left(l_{0}-r\right)+r \chi
\end{aligned}
$$

The denotation used above are as follows: $E_{i}$ - Young's modulus [GPa], $J_{i}-$ moment of inertia [m ${ }^{4}$ ], $A_{i}-\mathrm{cross}$ sectional area $\left[\mathrm{m}^{2}\right], \rho_{i}$ - material density $\left[\mathrm{kg} / \mathrm{m}^{3}\right], C_{H, L}$ - rotational spring stiffness $[\mathrm{Nm}], P-$ external load $[\mathrm{N}]$, $W_{i}$ - transversal displacement [m], $U_{i}$-longitudinal displacement [m], $R$-loading head radius [m], $r$-load receiving head radius $[\mathrm{m}], m$ - mass of heads $[\mathrm{kg}], I_{0}$-transom length $[\mathrm{m}]$.

On the basis of the Hamilton's principle, on which one performs all required mathematical operations one obtains equations of motion:

$E_{i} J_{i} \frac{\partial^{4} W_{i}\left(x_{i}, t\right)}{\partial x_{i}^{4}}-E_{i} A_{i} \frac{\partial}{\partial}\left[\left[\frac{\partial U_{i}\left(x_{i}, t\right)}{\partial x_{i}}+\frac{1}{2}\left(\frac{\partial W_{i}\left(x_{i}, t\right)}{\partial x_{i}}\right)^{2}\right] \frac{\partial W_{i}\left(x_{i}, t\right)}{\partial x_{i}}\right]+\rho_{i} A_{i} \frac{\partial^{2} W_{i}\left(x_{i}, t\right)}{\partial t^{2}}=0, i=1-4$

The compressive internal force is defined as:

$S_{i}(t)=-E_{i} A_{i}\left[\frac{\partial U_{i}\left(x_{i}, t\right)}{\partial x_{i}}+\frac{1}{2}\left[\frac{\partial W_{i}\left(x_{i}, t\right)}{\partial x_{i}}\right]^{2}\right]$

while longitudinal displacement as:

$U_{i}\left(x_{i}, t\right)=-\frac{S_{i}(t) x_{i}}{E_{i} A_{i}}-\frac{1}{2} \int_{0}^{x_{i}}\left[\frac{\partial W_{i}\left(x_{i}, t\right)}{\partial x_{i}}\right]^{2} d x_{i}$

The full set of geometrical as well as natural boundary conditions can be written in the following form:

$\left.W_{l}\left(x_{1}, t\right)\right|_{x l=0}=0,\left.\frac{\partial W_{1}\left(x_{1}, t\right)}{\partial x_{I}}\right|_{x l=0}=0,\left.W_{2}\left(x_{2}, t\right)\right|_{x 2=0}=0,\left.\frac{\partial W_{2}\left(x_{2}, t\right)}{\partial x_{2}}\right|_{x 2=0}=0$

$W_{2}\left(x_{2}, t\right)^{x_{2}=l_{2}}=\left.W_{3}\left(x_{3}, t\right)\right|_{x 3=0}, W_{3}\left(x_{3}, t\right)^{x_{3}=l_{3}}=\left.W_{4}\left(x_{4}, t\right)\right|_{x 4=0}$

$W_{1}\left(x_{1}, t\right)^{x_{I}=l_{l}}=W_{4}\left(x_{4}, t\right)^{x_{4}=l_{4}},\left.\frac{\partial W_{1}\left(x_{1}, t\right)}{\partial x_{1}}\right|^{x_{1}=l_{l}}=\left.\frac{\partial W_{4}\left(x_{1}, t\right)}{\partial x_{4}}\right|^{x_{4}=l_{4}}$ 
$-\left.E_{3} J_{3} \frac{\partial^{2} W_{3}\left(x_{3}, t\right)}{\partial x_{3}^{2}}\right|_{x_{3}=0}+C_{L}\left(\left.\frac{\partial W_{3}\left(x_{3}, t\right)}{\partial x_{3}}\right|_{x_{3}=0}-\left.\frac{\partial W_{2}\left(x_{2}, t\right)}{\partial x_{2}}\right|^{x_{2}=l_{2}}\right)=0$

$\left.E_{2} J_{2} \frac{\partial^{2} W_{2}\left(x_{2}, t\right)}{\partial x_{2}^{2}}\right|^{x_{2}=l_{2}}-C_{L}\left(\left.\frac{\partial W_{3}\left(x_{3}, t\right)}{\partial x_{3}}\right|_{x_{3}=0}-\left.\frac{\partial W_{2}\left(x_{2}, t\right)}{\partial x_{2}}\right|^{x_{2}=l_{2}}\right)=0$

$-\left.E_{4} J_{4} \frac{\partial^{2} W_{4}\left(x_{4}, t\right)}{\partial x_{4}^{2}}\right|_{x_{4}=0}+C_{H}\left(\left.\frac{\partial W_{4}\left(x_{4}, t\right)}{\partial x_{4}}\right|_{x_{4}=0}-\left.\frac{\partial W_{3}\left(x_{3}, t\right)}{\partial x_{3}}\right|^{x_{3}=I_{3}}\right)=0$

$\left.E_{3} J_{3} \frac{\partial^{2} W_{3}\left(x_{3}, t\right)}{\partial x_{3}^{2}}\right|_{x_{3}=0}-C_{H}\left(\left.\frac{\partial W_{4}\left(x_{4}, t\right)}{\partial x_{4}}\right|_{x_{4}=0}-\left.\frac{\partial W_{3}\left(x_{3}, t\right)}{\partial x_{3}}\right|^{x_{3}=l_{3}}\right)=0$

$\left.E_{2} J_{2} \frac{\partial^{3} W_{2}\left(x_{2}, t\right)}{\partial x_{2}^{3}}\right|^{x_{2}=l_{2}}+\left.S_{2} \frac{\partial W_{2}\left(x_{2}, t\right)}{\partial x_{2}}\right|^{x_{2}=l_{2}}-\left.E_{3} J_{3} \frac{\partial^{3} W_{3}\left(x_{3}, t\right)}{\partial x_{3}^{3}}\right|_{x_{3}=0}+\left.S_{3} \frac{\partial W_{3}\left(x_{3}, t\right)}{\partial x_{3}}\right|_{x_{3}=0}=0$

$\left.E_{3} J_{3} \frac{\partial^{3} W_{3}\left(x_{3}, t\right)}{\partial x_{3}^{3}}\right|^{x_{3}=l_{3}}+\left.S_{3} \frac{\partial W_{3}\left(x_{3}, t\right)}{\partial x_{3}}\right|^{x_{3}=l_{3}}-\left.E_{4} J_{4} \frac{\partial^{3} W_{4}\left(x_{4}, t\right)}{\partial x_{4}^{3}}\right|_{x_{4}=0}+\left.S_{4} \frac{\partial W_{4}\left(x_{4}, t\right)}{\partial x_{4}}\right|_{x_{4}=0}=0$

$\left.E_{I} J_{I} \frac{\partial^{2} W_{I}\left(x_{l}, t\right)}{\partial x_{I}^{2}}\right|^{x_{l}=l_{l}}+\left.E_{4} J_{4} \frac{\partial^{2} W_{4}\left(x_{4}, t\right)}{\partial x_{4}^{2}}\right|^{x_{4}=l_{4}}+P \frac{r-l_{0}}{R-r}\left(\left.\frac{\partial W_{I}\left(x_{1}, t\right)}{\partial x_{I}}\right|^{x_{l}=l_{l}}\left(R-l_{0}\right)+-\left.W_{I}\left(x_{l}, t\right)\right|^{x_{1}=l_{l}}\right)=0$

$\left.E_{I} J_{I} \frac{\partial^{3} W_{I}\left(x_{1}, t\right)}{\partial x_{I}^{3}}\right|^{x_{1}=l_{l}}+\left.E_{4} J_{4} \frac{\partial^{3} W_{4}\left(x_{4}, t\right)}{\partial x_{4}^{3}}\right|^{x_{4}=l_{4}}+P \frac{1}{R-r}\left(\left.\frac{\partial W_{l}\left(x_{l}, t\right)}{\partial x_{I}}\right|^{x_{l}=l_{l}}\left(R-l_{0}\right)+-\left.W_{I}\left(x_{l}, t\right)\right|^{x_{l}=l_{l}}\right)+$

$-\left.m \frac{\partial^{2} W_{I}\left(x_{l}, t\right)}{\partial t^{2}}\right|^{x_{1}=l_{l}}=0$

$S_{1}+S_{2}=P, S_{2}=S_{3}=S_{4}$

When the general description of the mathematical model of the column subjected to the specific load is done, one can start the formulation of the residual force generated by the piezorod. It is assumed that the column keeps the rectilinear form of static equilibrium and that the voltage is being applied in direction perpendicular to the axis of the column. At this stage one defines the potential energy in the following form:

$E_{p}=\frac{1}{2} \sum_{i=1}^{4} \int_{\Omega_{i}} \sigma_{x i} \varepsilon_{x i} d \Omega_{i}-\frac{1}{2} \int_{\Omega_{3}} D_{z} E_{z} d \Omega_{3}$

where

$\sigma_{x i}=E_{i} \varepsilon_{x i}\left(x_{i}\right) i=1,2,4 \quad \sigma_{x 3}=E_{3} \varepsilon_{x 3}\left(x_{3}\right)-e_{31} E_{z}$

$\varepsilon_{x i}=\frac{d U_{i}\left(x_{i}\right)}{d x_{i}}$

$D_{z}=e_{31} \varepsilon_{x 3}\left(x_{3}\right)+\psi_{33} E_{z}$ 
$E_{z}$ - electirical filed, $D_{z}$ - electrical displacement, $\varepsilon_{x i}$ - strain, $\sigma_{x i}$ - stress, $e_{31}$ - piezoelectric constant, $\psi_{33}$ - piezo conductivity.

Introduction of Eqs. 26-29 into Eq. 25 and definition of electric field as

$$
E_{z}=V_{T} / h
$$

as well as piezoelectric force as

$$
F_{p}=-b e_{31} V_{T}
$$

(where: $V_{T}$ - electrical field voltage, $h$ piezo height, $b$ - piezo width) allows one to write:

$$
E_{p}=\frac{1}{2} \sum_{i=1}^{4} E_{i} A_{i} \int_{0}^{l_{i}}\left[\frac{d U_{i}\left(x_{i}\right)}{d x_{i}}\right]^{2} d x_{i}+F_{p} \int_{0}^{l_{3}} \frac{d U_{3}\left(x_{3}\right)}{d x_{3}} d x_{3}-\frac{1}{2 h} b l_{3} \psi_{33} V_{T}^{2}
$$

Calculation of variation from Eq. 32 leads to the differential equations which are supplemented by boundary conditions for longitudinal displacements.

$\frac{d^{2} U_{i}\left(x_{i}\right)}{d x_{i}^{2}}=0$

$\left.E_{2} A_{2} \frac{d U_{2}\left(x_{2}\right)}{d x_{2}}\right|^{x_{2}=l_{2}}-\left.E_{3} A_{3} \frac{d U_{3}\left(x_{3}\right)}{d x_{3}}\right|_{x_{3}=0}-F_{p}=\left.0 \quad E_{3} A_{3} \frac{d U_{3}\left(x_{3}\right)}{d x_{3}}\right|^{x_{3}=l_{3}}-\left.E_{4} A_{4} \frac{d U_{4}\left(x_{4}\right)}{d x_{4}}\right|_{x_{4}=0}+F_{p}=0$

$\left.E_{1} A_{1} \frac{d U_{1}\left(x_{1}\right)}{d x_{1}}\right|^{x_{1}=l_{l}}+\left.E_{4} A_{4} \frac{d U_{4}\left(x_{4}\right)}{d x_{4}}\right|^{x_{4}=l_{4}}=\left.0^{\prime} U_{I}\left(x_{1}\right)\right|_{x_{1}=0}=\left.0^{\prime} U_{1}\left(x_{1}\right)\right|^{x_{1}=l_{l}}=\left.U_{4}\left(x_{4}\right)\right|^{x_{4}=l_{4}}$,

$\left.U_{2}\left(x_{2}\right)\right|^{x_{2}=l_{2}}=\left.\left.U_{3}\left(x_{3}\right)\right|_{x_{3}=0}{ }^{\prime} \quad U_{3}\left(x_{3}\right)\right|^{x_{3}=l_{3}}=\left.\left.U_{4}\left(x_{4}\right)\right|_{x_{4}=0} U_{2}\left(x_{2}\right)\right|_{x_{2}=0}=0$

Finally, one can write that the residual force is represented by the following relation:

$F=F_{p} \frac{E_{1} A_{1} E_{2} A_{2} E_{4} A_{4} l_{3}}{E_{3} A_{3} Q l_{2}+E_{2} A_{2}\left(Q l_{3}+E_{3} A_{3}\left(E_{4} A_{4} l_{1}+E_{1} A_{1} l_{4}\right)\right)}$

$Q=E_{1} A_{1} E_{4} A_{4}$

The residual force will lead to prestressing of the investigated column and must be introduced into equations of motion:

$E_{i} J_{i} \frac{\partial^{4} W_{i}\left(x_{i}, t\right)}{\partial x_{i}^{4}}-\left(S_{i}(t) \pm F_{i}\right) \frac{\partial^{2} W_{i}\left(x_{i}, t\right)}{\partial x_{i}^{2}}+\rho_{i} A_{i} \frac{\partial^{2} W_{i}\left(x_{i}, t\right)}{\partial t^{2}}=0 i=1-4$

$\left|F_{i}\right|=|F|$

On the basis of the described mathematical model one will present an influence of the prestressing on the stability and vibrations of the studied system. 


\section{NUMERICAL SIMULATIONS}

The results are presented in the non-dimensional form with the use of the following relations:

$p=\frac{P l^{2}}{E_{l} J_{l}+E_{2} J_{2}}, f=\frac{F l^{2}}{E_{l} J_{l}+E_{2} J_{2}}, c_{L}=\frac{C_{L} l}{E_{l} J_{l}+E_{2} J_{2}}, c_{H}=\frac{C_{H} l}{E_{l} J_{l}+E_{2} J_{2}}$,

$r_{2 l}=\frac{E_{2} J_{2}}{E_{1} J_{1}}, r_{32}=\frac{E_{3} J_{3}}{E_{2} J_{2}}, r_{43}=\frac{E_{4} J_{4}}{E_{3} J_{3}}, k_{i}=\frac{\left(S_{i} \pm F_{i}\right) l^{2}}{E_{1} J_{1}+E_{2} J_{2}}$,

$\zeta_{A}=\frac{R}{l_{l}}, \zeta_{B}=\frac{r}{R}, \zeta_{C}=\frac{l_{0}}{R}, \zeta_{D}=\frac{m}{\left(\rho_{1} A_{1}+\rho_{2} A_{2}\right) l}$,

$(40 \mathrm{a}-0)$

$\omega=\sqrt{\Omega^{2} \frac{\left(\rho_{1} A_{1}+\rho_{2} A_{2}\right) l^{4}}{E_{1} J_{1}+E_{2} J_{2}}}, d_{2}=\frac{l_{2}}{l}, d_{3}=\frac{l_{3}}{l}$

The results are focused on the characteristic curves, vibration modes, shape of the buckling envelopes as well as loading capacity curves.

The discussed in this paper instability is devoted into local or global. The creation of those regions is done on the basis of comparison of the bifurcation load of the non-linear system (Figure 1) and the critical load of the corresponding linear one. The linear column is defined as one composed only of external member (rod 1) in relation to the structure from the Figure 1 . The linear system is marked in all results as $K L$. In the case of the linear system the bifurcation load equals to the critical one. It was assumed that $E_{1} J_{1}+E_{2} J_{2}=$ const and one have calculated cross-sectional areas of rods 1 and 2. Due to presence of the fixed support as well as structure of the non-linear system the liner one used in this paper can also be used in studies done by Tomski and Uzny (2013).

The local instability occurs when the critical load of the linear system $K L$ is greater than bifurcation load of the nonlinear system (multi-member). In the opposite situation the global instability occurs. The studies on local and global instability were also presented by Tomski and Szmidla (2003), Uzny (2011), Uzny and Sokół (2014). The results from this paper (which are obtained on the basis of an analytical solution) can be verified during the experimental studies.

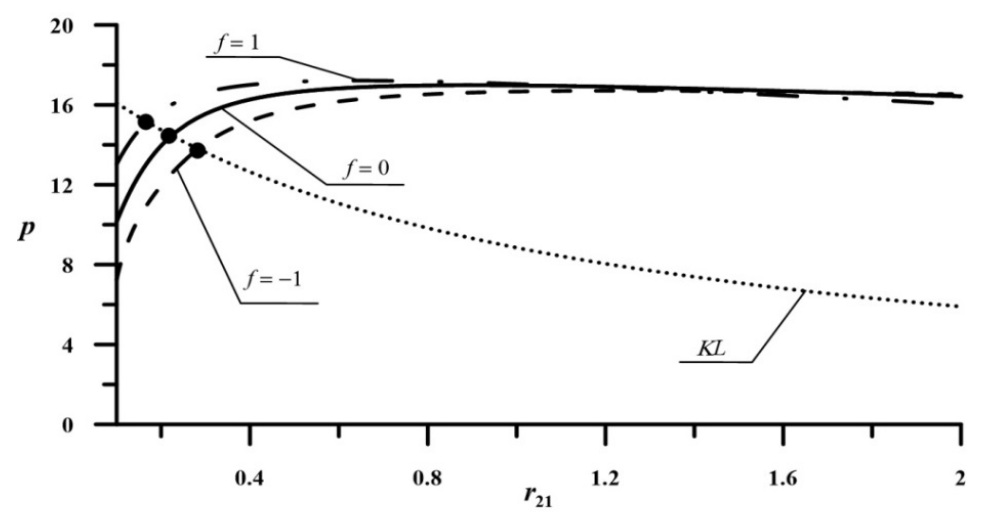

Figure 2: Instability regions at different magnitude of the prestressing force, $\left(\zeta_{\mathrm{A}}=0.2, \zeta_{\mathrm{B}}=0.2, \zeta_{\mathrm{C}}=0.5, r_{32}=r_{43}=1, d_{2}=0.25, d_{3}=\right.$ $\left.0.2, c_{H}=5, c_{\mathrm{L}}=5\right)$

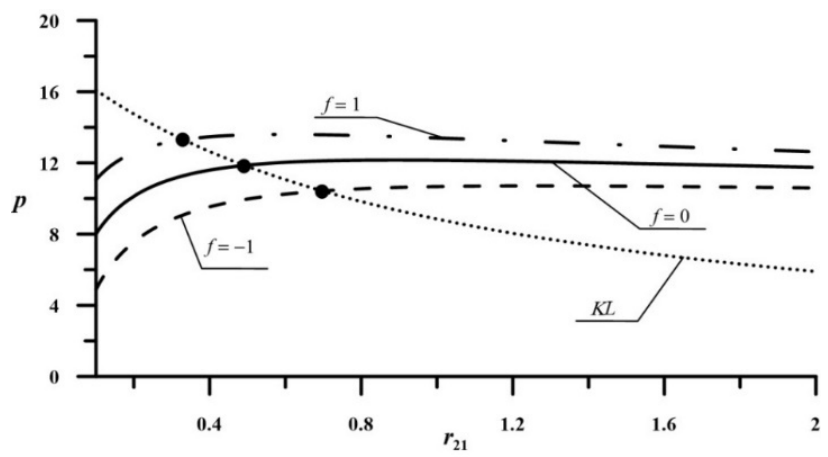

Figure 3: Instability regions at different magnitude of the prestressing force, $\left(\zeta_{A}=0.2, \zeta_{B}=0.2, \zeta_{C}=0.5, r_{32}=r_{43}=1, d_{2}=0.25, d_{3}=\right.$ $0.2, c_{\mathrm{H}}=0.5, c_{\mathrm{L}}=5$ ) 
As presented in the Figures 2 and 3 an appliance of the voltage to the piezorod one can result in increase or reduction of the loading capacity of the non-linear system, what at the same time leads to the change of the instability regions. The stiffer the connections of piezorod to the host structure the smaller influence of the prestressing on the size of the instability regions. Moreover, the smaller the bending rigidity ratio $r_{21}$ magnitude the greater change in the loading capacity can be observed during prestressing. Black dots mark the boundary magnitude of bending rigidity ratio $r_{21}$ and separate the local instability region from the global one. The greatest magnitude of the critical load is found at the lowest magnitude of the $r_{21}$. It has also been noticed that prestressing in combination with an increase in bending rigidity ratio may finally lead to the decrease of the bifurcation load in relation to the unprestressed system.

Along with the difference in the shape of the loading capacity curves one can present characteristic curves plotted in the external load - vibration frequency plane. The Figures 4-6 are plotted at different magnitude of the bending rigidity ratio $r_{21}=$ $0.4,1$ and 1.6. The continuous line corresponds to the unprestressed system while the others represent the prestressing force $f= \pm 2$. It has been noticed that in relation to the direction of the electric field vector one can obtain the control of the shape of the characteristic curve regardless to the parameters of the system. At the beginning (at small magnitude of the external load) all presented curves are overlapping each other up to the point in which the greatest magnitude of the vibration frequency for each curve can be found. Above that point curves are separated and change the inclination angle. As presented the $+f$ force gives smaller change in the shape of the characteristic curve in relation to the $-f$ one.

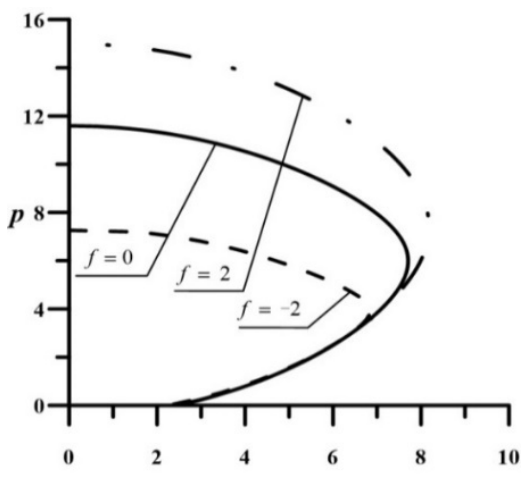

Figure 4: Characteristic curves, $\left(\zeta_{A}=0.2, \zeta_{B}=0.2, \zeta_{C}=0.5, \zeta_{D}=0.2, r_{21}=0.4, r_{32}=r_{43}=1, d_{2}=0.25, d_{3}=0.2, c_{H}=0.5, c_{L}=5\right)$

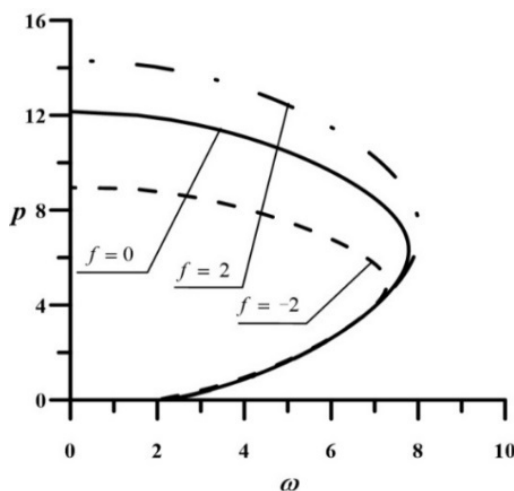

Figure 5: Characteristic curves, $\left(\zeta_{\mathrm{A}}=0.2, \zeta_{\mathrm{B}}=0.2, \zeta_{\mathrm{C}}=0.5, \zeta_{\mathrm{D}}=0.2, r_{21}=r_{32}=r_{43}=1, d_{2}=0.25, d_{3}=0.2, c_{\mathrm{H}}=0.5, c_{\mathrm{L}}=5\right)$

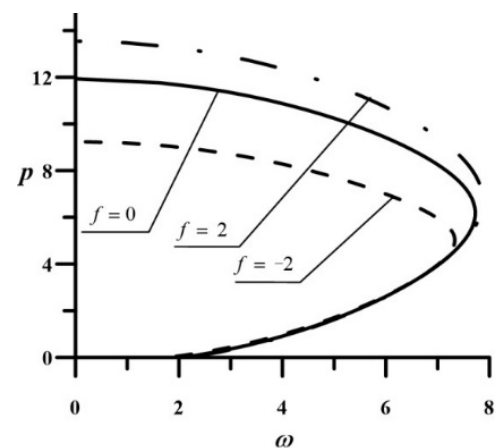

Figure 6: Characteristic curves, $\left(\zeta_{A}=0.2, \zeta_{B}=0.2, \zeta_{C}=0.5, \zeta_{D}=0.2, r_{21}=1.6, r_{32}=r_{43}=1, d_{2}=0.25, d_{3}=0.2, c_{H}=0.5, c_{L}=5\right)$ 
Table 1 Vibration modes $\left.\zeta_{A}=0.2, \zeta_{B}=0.2, \zeta_{C}=0.2, \zeta_{D}=0.2, r_{32}=r_{43}=1, d_{2}=0.25, d_{3}=0.5, c_{L}=1, c_{H}=0.1\right)$

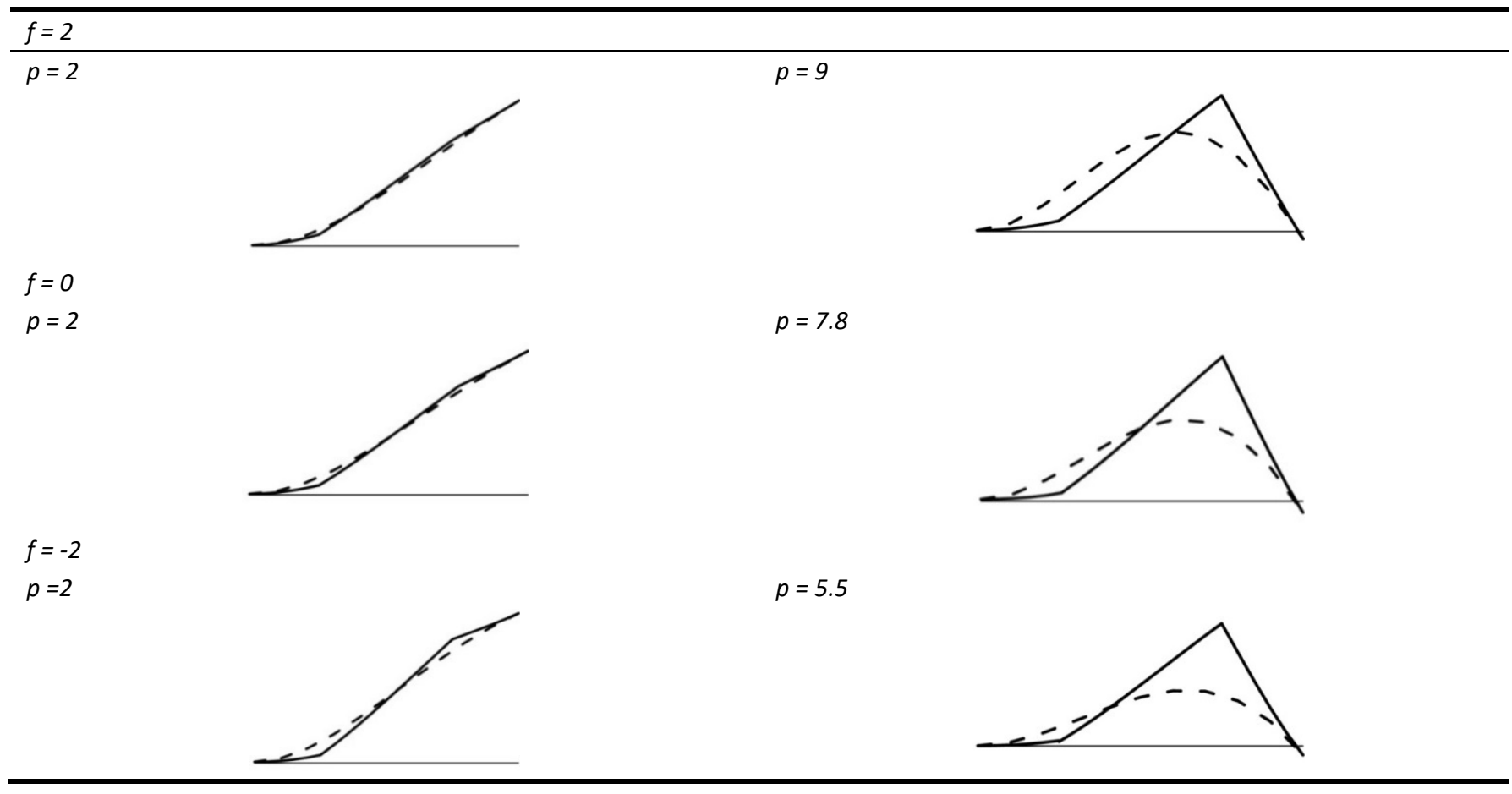

In Table 1 the vibration modes at different level of prestressing are plotted (continuous line - member I, dashed line member II). In each row the table contains two vibration modes, one calculated at the positive slope of the characteristic curve while the other at negative one. This phenomenon is characteristic when the divergence-pseudoflutter systems are used. The presence of two vibration modes was discussed and presented by Tomski and Uzny (2013). The generation of $+f$ force causes that bent axes of rods are placed closer to each other in relation to $f=0$ case at much greater magnitude of the external load. At $-f$ the difference of the bent axes shape is the greatest at the lowest $p$.

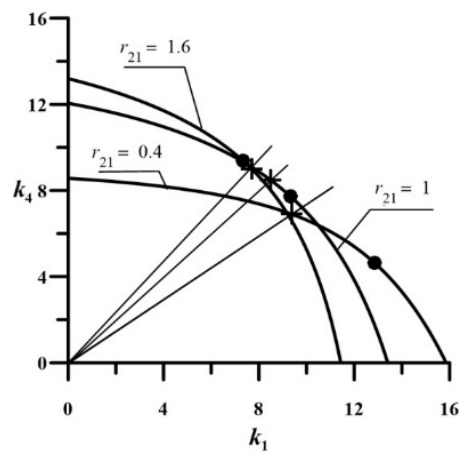

Figure 7: Buckling envelopes at different bending rigidity ratio $\left(\zeta_{\mathrm{A}}=0.2, \zeta_{\mathrm{B}}=0.2, \zeta_{\mathrm{C}}=0.5, r_{32}=r_{43}=1, d_{3}=0.2, c_{\mathrm{H}}=5, c_{\mathrm{L}}=5\right)$

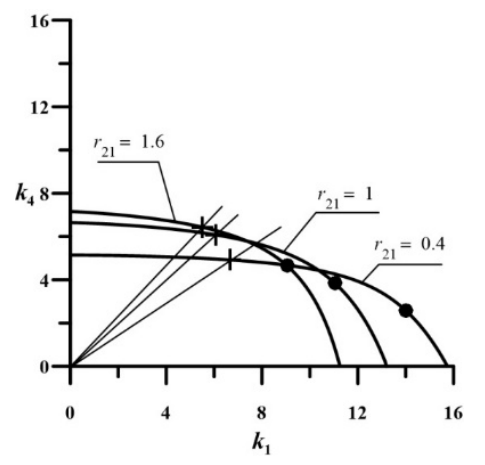

Figure 8: Buckling envelopes at different bending rigidity ratio $\left(\zeta_{\mathrm{A}}=0.2, \zeta_{\mathrm{B}}=0.2, \zeta_{\mathrm{C}}=0.5, r_{32}=r_{43}=1, d_{3}=0.2, c_{\mathrm{H}}=0.5, c_{\mathrm{L}}=5\right)$ 


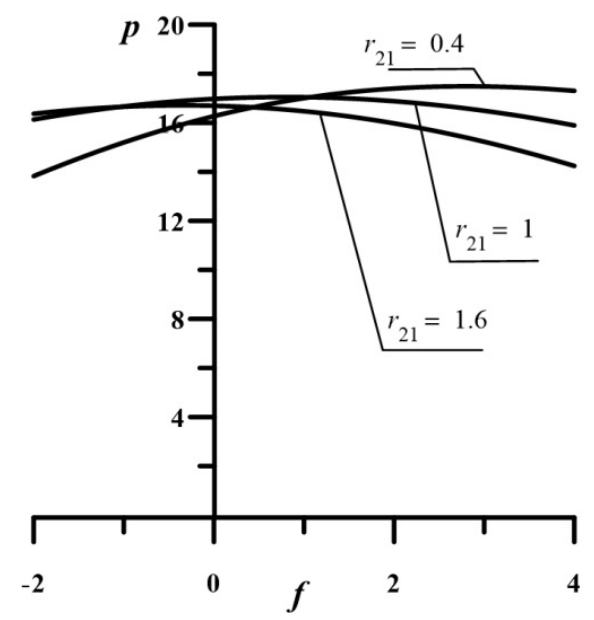

Figure 9: Influence of the prestressing on bifurcation load at different bending rigidity ratio $\left(\zeta_{A}=0.2, \zeta_{B}=0.2, \zeta_{C}=0.5\right.$, $\left.r_{32}=r_{43}=1, d_{2}=0.25, d_{3}=0.2, c_{H}=5, c_{L}=5\right)$

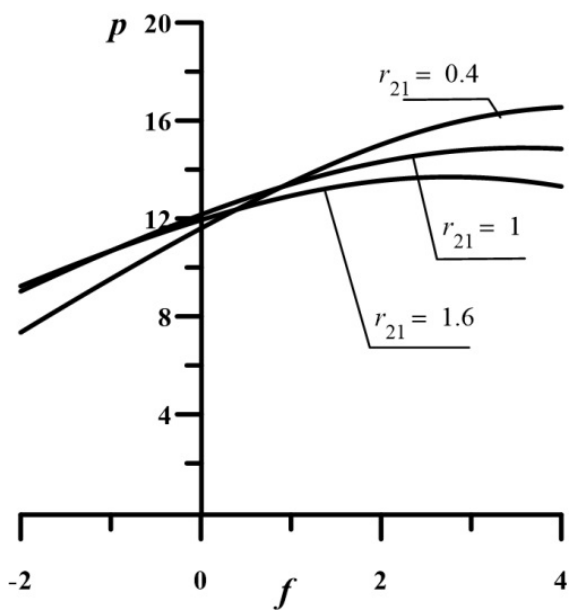

Figure 10: Influence of the prestressing on bifurcation load at different bending rigidity ratio $\left(\zeta_{A}=0.2, \zeta_{B}=0.2, \zeta_{C}=0.5\right.$,

$$
r_{32}=r_{43}=1, d_{2}=0.25, d_{3}=0.2, c_{H}=0.5, c_{L}=5 \text { ) }
$$

On the basis of the curves $p-r_{21}$ one have plotted buckling envelopes (Figures 7,8 ) on which the critical loads (black dots) as well as bifurcation loads (cross marks) have been placed. The buckling envelopes are presented on the plane: internal force of external member - internal one. The greater the distance between the cross and the dot the greater increase in loading capacity can be obtained. It has been found that at infinite stiffness of the rotational springs the bifurcation and critical points are place on the line having start in $(0,0)$ point and inclined at $\operatorname{arctg}\left(\frac{E_{2} A_{2}}{E_{1} A_{1}}\right)$ angle. As a supplementary material the Figures 9 and 10 are introduced where one can find bifurcation load - prestressing force relationship curves. It has be noticed that regardless to magnitude of the bending rigidity ratio one can obtain the correction of the shape of the loading curves. The rapidness of this correction depends not only on magnitude of the prestressing force but also on other parameters of the system.

\section{CONCLUSION}

This paper covers the problem of control of loading capacity and vibration frequency of a column subjected to the specific load realized by circular elements of heads. The control has been achieved with the use of the piezoceramic rod integrated with the host structure.

On the basis of the results of numerical simulations one can conclude that:

- $\quad$ the application of voltage to the piezorod creates compressive or tensile force in relation to the electric field vector and leads to control of all investigated parameters like loading capacity, instability regions and vibration frequency,

- the area of control depends on applied voltage as well as parameters of the host structure, 
an influence of the prestressing on the vibration frequency increases with an increase in external load magnitude (the characteristic curves at low magnitude of external load are overlapping each other),

Elements made of piezoceramic material can fully satisfy the problem of instability and vibration control. The studies on the presented in this paper phenomenon should be extended to take into account more factors like parameters of the heads or length relationship between rods.

Author Contributions: Work concept, numerical simulations, results validation, writing K Sokół.

Editor: Jan Awrejcewicz.

\section{References}

Adali, S., Sadek, I.S., Bruch, J.C., (2005). Optimization of composite plates with piezoelectric stiffener-actuators under in-plane compressive loads. Composite Structures 71(3-4):293-301.

Bendine, K., Wankhade, L.(2017). Optimal shape control of piezolaminated beams with different boundary condition and loading using genetic algorithm. International Journal of Advanced Structural Engineering 375-384):4(9.

Faria, A.R., (2004). On buckling enhancement of laminated beams with piezoelectric actuators via stress stiffening. Composite Structures 65(2):187-192.

Irschik, H., (2002). A review on static and dynamic shape control of structures by piezoelectric actuation. Engineering Structures 24(1):5-11.

Kozupa, M., Wiciak, J., (2010). Active vibration control of rectangular plate with distributed piezoelements excited acoustically and mechanically. Acta Physica Polonica A 118. DOI: 10.12693/APhysPolA.118.95.

Mukherjee, A., Saha Chaudhuri, A., (2002). Active control of dynamic instability of piezolaminated imperfect columns. Smart Materials and. Structures 11:874-879.

Mukherjee, A., Saha Chaudhuri, A., (2004). Exact solutions for instability control of piezolaminated imperfect struts. AIAA Journal 42:857-859.

Peng, J., Wang, XY., Xie, XZ., (2019). Vibration control for the nonlinear resonant response of a piezoelectric elastic beam via time-delayed feedback. Smart Materials and Structures 28(9).

Przybylski, J., Sokół K., (2011). Shape control of an eccentrically loaded column by means of a piezoceramic rod. Thin - Walled Structures 49: 652-659.

Shakeri Mobarakeh, P., Grinchenko, V.T., Sotannia, B., (2019). Bending vibrations of bimorph piezoceramic plates of noncanonical shape. International Applied Mechanics 55:321-331.

Sokół, K., Uzny S., (2015). Linear and nonlinear vibrations control of a two member column by means of piezoceramic element. AIP Conference Proceedings 1648, DOI: 10.1063/1.4913076.

Sokół, K., (2019). Instability and vibration control by means of piezoceramic element. Theoretical Approaches in Non-Linear Dynamical Systems: 485-497.

Song, G., Sethi, V., Li H.N., (2006).Vibration control of civil structures using piezoceramic smart materials: A review. Engineering Structures 28 (11):1513-1524.

Thompson, S., Loughlan, J., (1995). The active buckling control of some composite column strips using piezoceramic actuators. Composite Structures 32:59-67.

Tomski, L., Szmidla, J., (2003). Local and global instability and vibration of overbraced Euler's column. Journal of Theoretical and Applied Mechanics 41: 137-154.

Tomski, L., Uzny, S., (2013). Vibrations and stability of a column subjected to the specific load realized by circular elements of heads. Mechanics and Mechanical Engineering 17:197 - 206. 
Uzny, S. (2011). Local and global instability and vibrations of a slender system consisting of two coaxial elements, Thin-Walled Structures. 49:618-626.

Uzny, S., Sokół, K., (2014). The regions of local and global instability of a two-member slender system with crack. Machine Dynamics Research. 38:75-83.

Wankhade L., Bajoria M, (2017) Numerical optimization of piezolaminated beams under static and dynamic excitations. Journal of Science: Advanced Materials and Devices 2(2):255-262.

Wankhade, L., Bajoria, M., (2019). Vibration analysis of piezolaminated plates for sensing and actuating applications under dynamic excitation. International Journal of Structural Stability and Dynamics19 (10).

Yang, ZX., He, XT., Peng, DD., Sun, JY., (2020). Free damping vibration of piezoelectric cantilever beams: a biparametric perturbation solution and its experimental verification. Applied Sciences-Basel 10(1).

Zenz, G., Humer, A., (2015). Stability enhancement of beam-type structures by piezoelectric transducers: theoretical, numerical and experimental investigations. Acta Mechanica 226(12):3961-3976. 